Technological University Dublin ARROW@TU Dublin

\title{
The running performance profile of elite gaelic football match- play
}

\author{
Shane Malone \\ Technological University Dublin, Shane.Malone@TUDublin.ie \\ Barry Solan \\ Technological University Dublin \\ Kieran Collins \\ Technological University Dublin, kieran.collins@tudublin.ie
}

Follow this and additional works at: https://arrow.tudublin.ie/ittsciart

Part of the Sports Sciences Commons

\section{Recommended Citation \\ Malone S, Solan B, Collins K. The Running Performance Profile of Elite Gaelic Football Match-Play. J Strength Cond Res. 2017 Jan;31(1):30-36. doi: 10.1519/JSC.0000000000001477. PMID: 27191694.}

This Article is brought to you for free and open access by the School of Science and Computing at ARROW@TU Dublin. It has been accepted for inclusion in Articles by an authorized administrator of ARROW@TU Dublin. For more information, please contact arrow.admin@tudublin.ie, aisling.coyne@tudublin.ie, gerard.connolly@tudublin.ie.

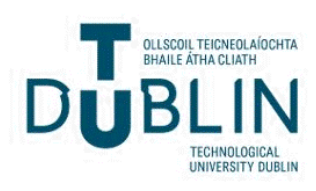




\title{
The Running Performance Profile of Elite Gaelic Football Match-Play
}

\author{
Shane Malone, ${ }^{1,2}$ Barky Solan, ${ }^{2}$ and Kieran Collins ${ }^{2}$ \\ ${ }^{1}$ Research Institute for Sport and Exercise Sciences, Liverpool John Moores University, Liverpool, United Kingdom; and \\ ${ }^{2}$ Department of Science, Gaelic Sports Research Centre, Institute of Technology Tallaght, Dublin, Ireland
}

\begin{abstract}
Malone, S, Solan, B, and Collins, K. Title: The running performance profile of elite Gaelic football match-play. J Strength Cond Res 31(1): 30-36, 2017-The current study examined (a) the match running performance of Gaelic football and (b) the decrement in match running performance with respect to position. Global positioning satellite system technologies (4-Hz; VX Sport) were used with 3 elite intercounty teams across 3 full seasons with 250 full game data sets collected. Game movements were classified according to game actions and distance covered across speed zone thresholds (total distance [TD], high-speed running distance [HSRD; $\geq 17 \mathrm{~km} \cdot \mathrm{h}^{-1}$ ], sprint distance [SD; $\geq 22 \mathrm{~km} \cdot \mathrm{h}^{-1}$ ]; accelerations [n]; peak speed $\left.\left[\mathrm{km} \cdot \mathrm{h}^{-1}\right]\right)$. The influence of running performance in each quarter on the subsequent quarter was analyzed across all positional roles. The mean $( \pm S D)$ TD and HSRD covered during the game were $8,889 \pm 1,448 \mathrm{~m}$ and $1,596 \pm 594 \mathrm{~m}$, respectively. Results show a temporal profile for TD with reductions in the second $(-4.1 \%)$, third $(-5.9 \%)$ and fourth $(-3.8 \%)$ quarters, respectively. There was a significant reduction in HSRD in the second $(-8.8 \%)$, third $(-15.9 \%)$, and fourth $(-19.8 \%)$ quarters when compared to the first quarter $(p<0.001)$. Positional differences were observed for distance-based measures with the middle 3 positions (half-back, midfield, and half-forward) completing the highest running performances. These positions also showed increased decrements in TD and HSRD and SD across quarters. The current data indicate a reduction in exercise intensity over the duration of elite Gaelic football match-play. It is unclear if this reduction is because of fatigue, pacing, contextual factors, or nutritional strategies employed by players.
\end{abstract}

KEY WORDS match analysis, high-speed running, team sport, intermittent exercise

Address correspondence to Shane Malone, shane.malone@mymail. ittdublin.ie.

31(1)/30-36

Journal of Strength and Conditioning Research

(C) 2016 National Strength and Conditioning Association

\section{INTRODUCTION}

$\mathrm{G}$ aelic football is one of the national sports of Ireland. Elite-level games are often played in front of attendances of 80,000 people and television pictures are broadcast around the world (23). The game is played with 2 teams of 15 players with the primary objective of both teams to outscore one another across the 70-minute period of competitive play (2). The game is best described as an invasion field sport with repeated high intensity actions that are unstable and stochastic in nature (7). Few attempts have been made to describe the running performance of elite-level Gaelic football players $(7,14)$. As a consequence of the limited work undertaken, the running performance of elite-level players has been appraised based on sports that appear to have a similar running profile, e.g., soccer. With the pitch dimensions of Gaelic football being 33\% larger than soccer, the transfer of these demands to Gaelic football may fail to fully appreciate the true running performance of competitive match-play (23).

Keane et al. (14) examined match demands across 4 championship and 4 national league games using videobased time-motion analysis and identified the mean distance covered was $8,594 \pm 1,056 \mathrm{~m}$. The majority of the distance $(66 \%)$ was accounted for by low intensity activities, such as walking and jogging. Conversely, high-speed activity (striding and sprinting) accounted for $12.4 \%$ and $3.7 \%$ of distance covered, respectively. Interestingly, no decrement in running performance was evident between the first and second halves. Furthermore, positional profiles were identified for 3 positional lines with nonsignificant differences observed for defenders $(8,523 \pm 1,175$ $\mathrm{m})$, midfielders $(9,131 \pm 977 \mathrm{~m})$, and forwards $(8,490 \pm$ $673 \mathrm{~m}$ ). More recently, McErlean et al. (18) performed audio-based time-motion analysis across 40 elite matchplay profiles. The investigation found that the mean time spent completing high intensity activities ranged from 4.1 seconds for forwards to 6.8 seconds for midfielders. The study also reported that $15.9 \%$ of the game was completed at high speed. This ranged from $10.7 \%$ for forwards to $22.1 \%$ for midfielders. Work-to-rest ratios were lowest for midfielders $(1: 3.6 \pm 0.6)$ and highest for forwards $(1: 13.0 \pm 6.6)$. Similar to previous findings, there was 
no decrement observed for running performance across halves of play. Additionally, O'Donoghue and King (20) analyzed the activity profile university footballers. They reported longer mean high intensity activity durations (5.7 seconds vs. 3.2 seconds) and shorter recovery periods compared with premier league soccer games, with $51 \%$ of recovery between bouts less than 20 seconds. Furthermore, $8 \%$ of these recovery periods were reported to last $\geq 90$ seconds. Similar to previous investigations $(14,18)$, positional differences were communicated, with central players performing a $50 \%$ greater number of bursts. Interestingly, central players were reported to have shorter recovery periods than any of the other positional groups. These central players were also reported to spend more time of match-play performing high intensity activities when compared with other positional groups.

Many of the previous studies within Gaelic football have been conducted using manual, vision-based notation analysis to determine activity profiles. This methodology of analysis has previously been reported to be labor intensive and outdated (9). The advancement in technology now allows for game movements and running performance to be investigated through the use of global positioning satellite systems (GPS). These systems have been validated for use within intermittent field sports like Gaelic football $(5,10,13,16)$. Therefore, with the information provided in previous investigations within Gaelic football being focused on 3 specific positions (defenders, midfielders, forwards), there is a need to update these positional profiles to account for the 5 specific positional lines of Gaelic football (full-back, half-back, midfield, half-forward, and fullforward). Specific information pertaining to these positional lines may provide more practical relevance to current practitioners with regard to drill construction within the Gaelic football training process. Unfortunately, there is currently limited contemporary information describing the match running performance of elite Gaelic football match-play with respect to position. Therefore, the aims of the current study were to (a) describe the match running performance of elite Gaelic football players using GPS technology and (b) examine the decrement of running performance during elite match-play competition with respect to position. It was hypothesized that highspeed running performance would decrease across quarters of match-play, with the middle 3 positions experiencing the highest decrement in running performance across quarters of match-play.

\section{Methods}

\section{Experimental Approach to Problem}

The current observational study was designed to examine match-play running performance of elite Gaelic football players across quarters using portable GPS technology. Fifty intercounty elite Gaelic football players $(n=50)$ playing in division 1 and division 2 were analyzed across
3 full-competition seasons (February 2010-September 2013) resulting in 250 individual samples being collected $(n=250)$. All players were competing at national league (NFL) and All-Ireland championship level. Game data were only included if a full match (70 minutes) was completed. Data were classified according to position (fullback, half-back, midfield, half-forward, and full-forward). All competitive matches took place between 1400 and 2000 hours. Temperatures during match-play ranged from 10 to $22^{\circ} \mathrm{C}$. The GPS was used to determine specific running performance variables during elite Gaelic football match-play. All players were advised to maintain their normal diet, with special emphasis being placed on the intake of fluids and carbohydrates.

\section{Subjects}

Fifty elite male Gaelic footballers with a mean $( \pm S D)$ age (26 \pm 4 years), height $(183 \pm 7 \mathrm{~cm})$, weight $(79 \pm 7 \mathrm{~kg})$, and years on squad ( $5 \pm 3$ years), respectively, volunteered to participate in the study. Players were selected as they were members of the county's squad that season and therefore were deemed the best players in the county at the time of data collection. After ethical approval, participants attended an information evening where they were briefed about the purpose, benefits, and procedures of the study. Written informed consent and medical declaration were obtained from participants in line with the procedures set by the local institutions research ethics committee.

\section{Experimental Procedures}

Participants wore an individual GPS unit (Issue: 330a, Firmware: 4.01.1.0; VX Sport, Lower Hutt, New Zealand) sampling at $4-\mathrm{Hz}$ in a total of 30 games. The GPS unit (mass: $76 \mathrm{~g} ; 48 \mathrm{~mm} \times 20 \mathrm{~mm} \times 87 \mathrm{~mm}$ ) was encased within a protective harness between the player's shoulder blades in the upper thoracic-spine region. The device was activated and satellite lock established for a minimum of 15 minutes before the commencement of each match (15). The validity and reliability of this device has previously been communicated $(5,16)$. Specifically, Malone et al. (16) report the VX Sport GPS to be reliable during intermittent exercise. Test-retest (7 days apart) reliability was reported for total distance covered $(\mathrm{m})$, maximum speed $\left(\mathrm{km} \cdot \mathrm{h}^{-1}\right)$, and average speed $\left(\mathrm{km} \cdot \mathrm{h}^{-1}\right)$. Systematic differences were examined using a paired $t$ test on the test-retest data and revealed no significant differences for the total distance covered $(300.5 \pm 3.3 ; 303.6 \pm 5.6 \mathrm{~m})$, maximum speed $\left(23.9 \pm 1.9 ; 24.1 \pm 1.3 \mathrm{~km} \cdot \mathrm{h}^{-1}\right)$, and average speed $\left(10.2 \pm 1.0 ; 10.2 \pm 0.9 \mathrm{~km} \cdot \mathrm{h}^{-1}\right)$. The typical error $(\mathrm{TE} \pm$ $95 \%$ confidence interval $[\mathrm{CI}])$ was $0.84 \pm 0.3$ for total distance covered, $0.75 \pm 0.26$ for maximum speed, and $0.55 \pm$ 0.19 for average speed, respectively. The coefficient of variation $(\mathrm{CV} \% \pm 95 \% \mathrm{CI})$ was $1.0 \pm 0.4$ for the total distance covered, $4.2 \pm 1.5$ for maximum speed, and $4.4 \pm 1.5$ for average speed, respectively. 


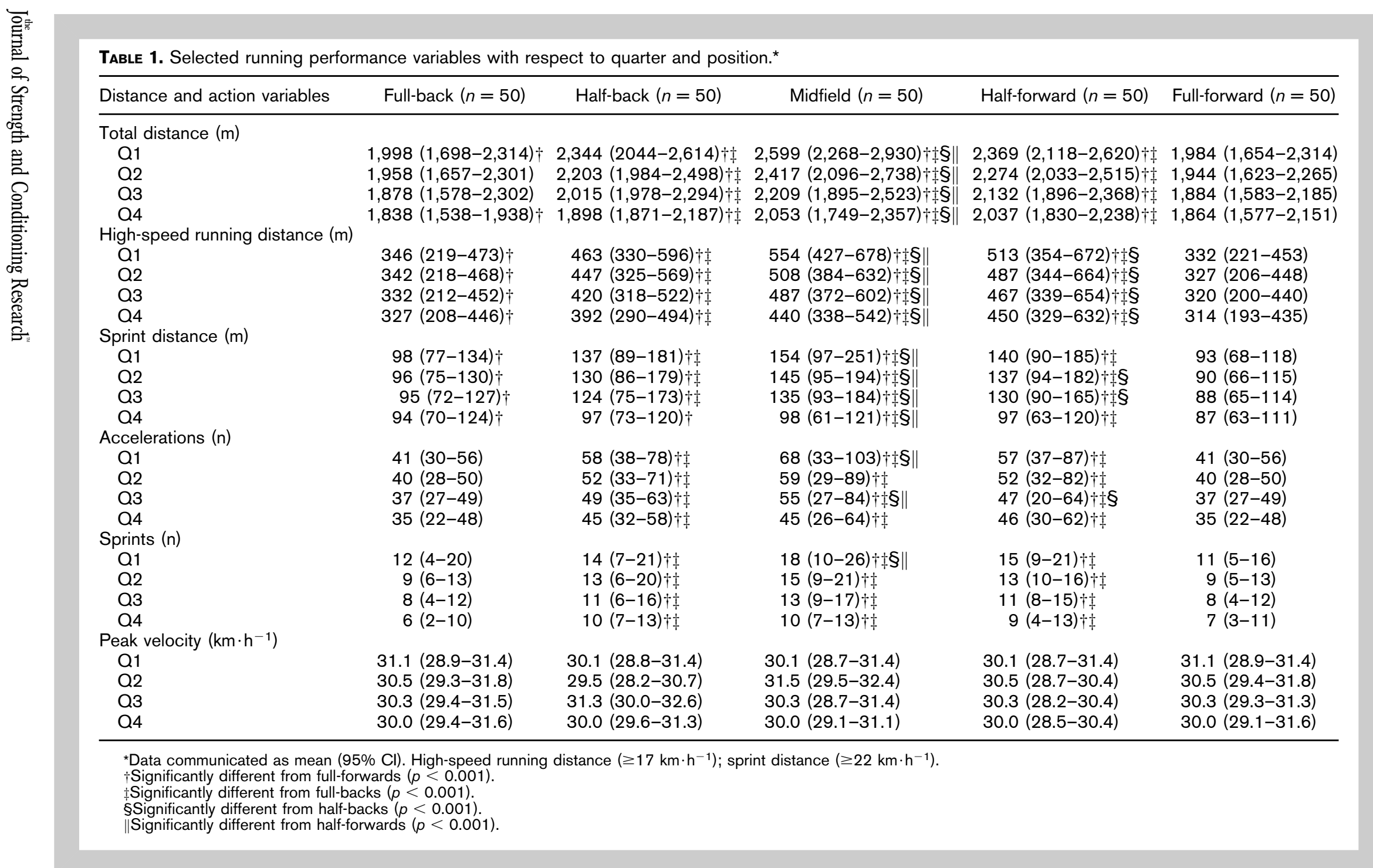




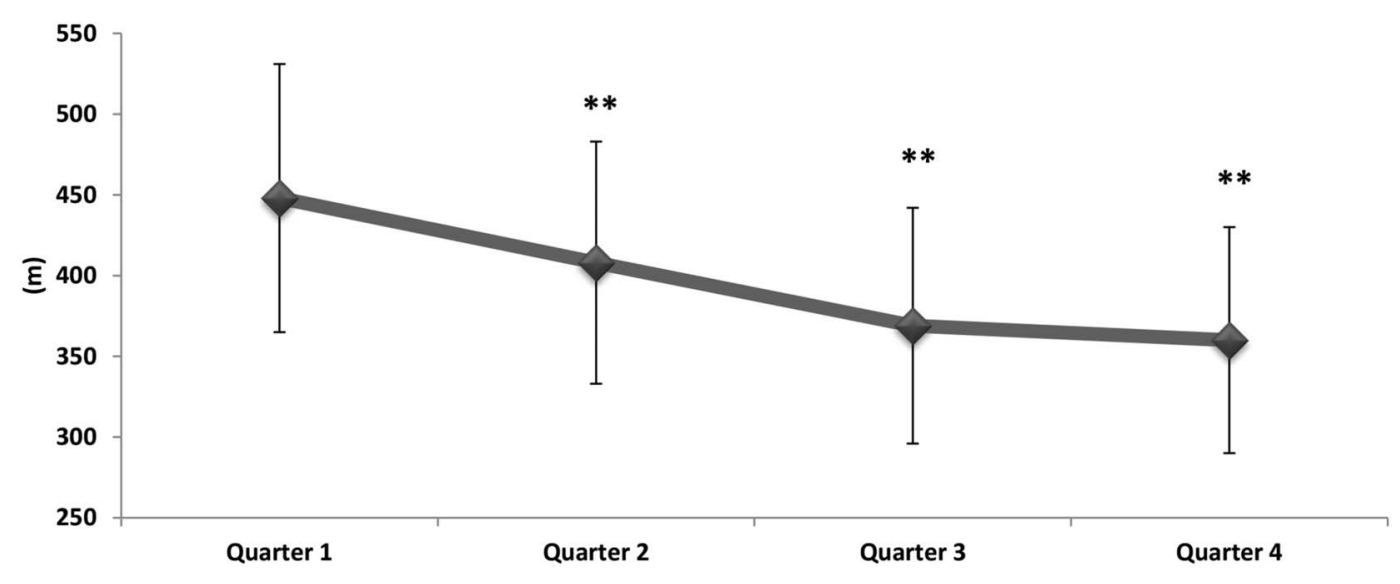

Figure 1. The change in high-speed running distance $\left(\mathrm{m} ; \geq 17 \mathrm{~km} \cdot \mathrm{h}^{-1}\right)$ across quarters of play during elite Gaelic football match-play $(n=250)$. Data reported as mean $\pm 95 \% \mathrm{Cl}$. ${ }^{*}$ Significantly different from first quarter (all $p<0.001$ ).

After each match, GPS data were downloaded using proprietary software (VX Sport). Each file was trimmed so that only data recorded when the player as on the field was included for further analysis. The proprietary software provided instantaneous raw velocity data at 0.25 -second intervals, which was then exported and placed into a customized Microsoft Excel spreadsheet (Microsoft, Redmond, WA, USA). The spreadsheet allowed analysis of distance covered $(\mathrm{m})$ in the following categories: total distance (TD), high-speed running distance (HSRD; $\geq 17 \mathrm{~km} \cdot \mathrm{h}^{-1}$ ), sprint distance $\left(\mathrm{SD} ; \geq 22 \mathrm{~km} \cdot \mathrm{h}^{-1}\right)$, accelerations $(\mathrm{n})$, and peak speed $\left(\mathrm{km} \cdot \mathrm{h}^{-1}\right)$. An acceleration effort was classified once a participant changed speed by $2 \mathrm{~km} \cdot \mathrm{h}^{-1}$ within 1 second of the given movement. The change was triggered over a minimum time of 2 seconds (to demarcate a lunge from a sprint). The acceleration stopped when the player decelerated to $<75 \%$ of maximum speed reached in the preceding acceleration effort $(7,17)$.

\section{Statistical Analyses}

The assumptions of normality were verified before parametric statistical analysis. All data are presented as means,

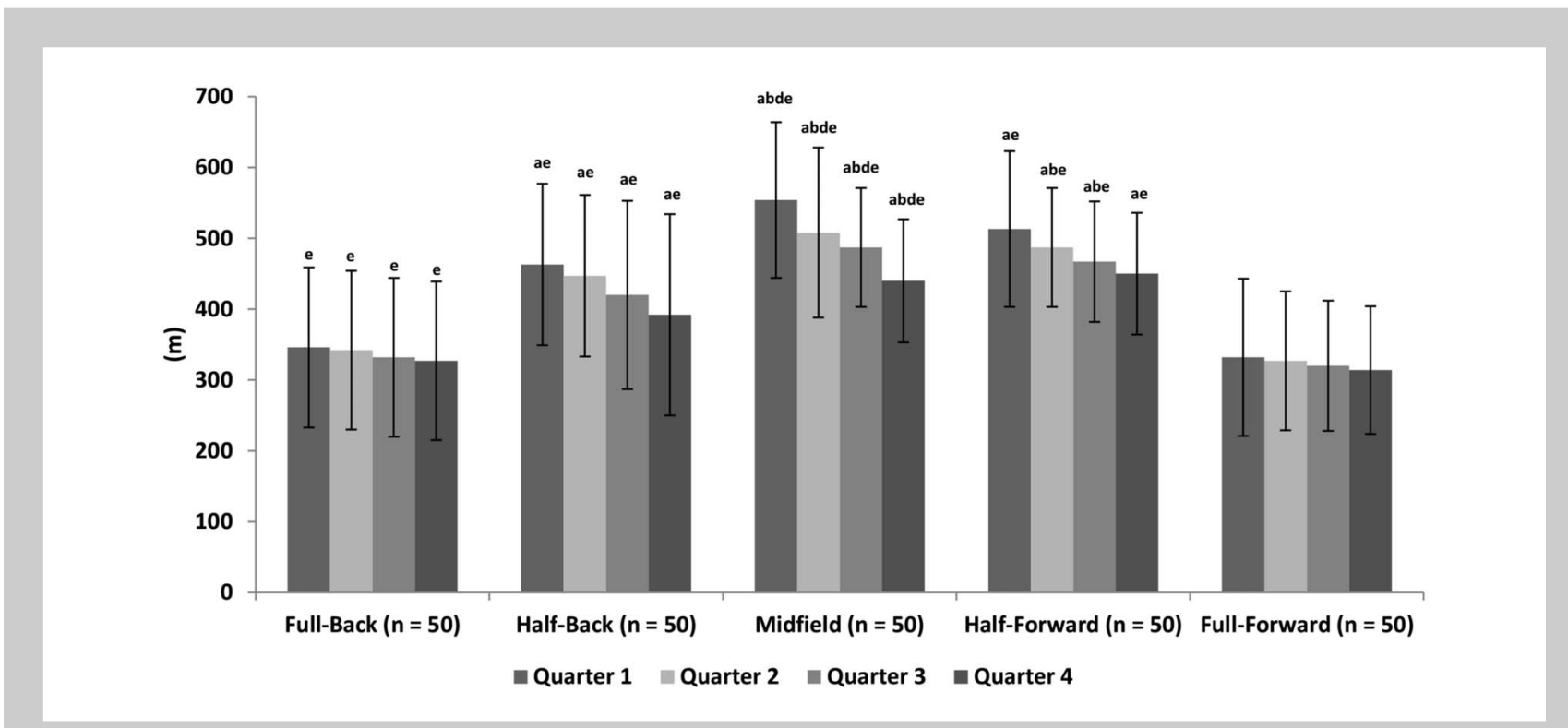

Figure 2. The changes in high-speed running distance $\left(\mathrm{m} ; \geq 17 \mathrm{~km} \cdot \mathrm{h}^{-1}\right)$ with respect to position across quarters of play. Data reported as mean $\pm 95 \%$ Cl. Significantly different from (a) full-backs, (b) half-backs, (c) midfielders, (d) half-forwards, (e) full-forwards (all $p<0.001$ ). 
standard deviations, and 95\% confidence intervals (95\% CI). A multivariate analysis of variance (MANOVA) was used to compare differences in running performance variables between positional groups (5) and quarter (3). The level of statistical significance was set at an accepted level of $p \leq$ 0.05 . When significant main effects were observed, Scheffe's post hoc test was applied. Standardized effect sizes (ES) were calculated with $<0.2,0.21-0.6,0.61-1.20,1.21-2.00$ and 2.01-4.0 representing trivial, small, moderate, large and very large differences, respectively (12). All statistical analyses were performed using SPSS for Windows (version 22; SPSS Inc., Chicago, IL, USA).

\section{Results}

Selected running performance variables are shown in Table 1 . The mean TD and HSRD covered during the game were $8,889 \pm 1,448$ and $1,596 \pm 594 \mathrm{~m}$, respectively. Players completed $181 \pm 39$ accelerations in addition to completing $445 \pm 69 \mathrm{~m}$ of $S D$ across $43 \pm 15$ sprints actions during competitive play with the peak speed achieved being $30.3 \pm 1.2 \mathrm{~km} \cdot \mathrm{h}^{-1}$. A significant main effect for quarter of play $(F=7.36, p<0.001)$ was observed. Post hoc analyses revealed that there were significant reductions in TD in the second $(\mathrm{ES}=0.35)$, third $(\mathrm{ES}=0.45)$, and fourth $(\mathrm{ES}=0.39)$ quarters when compared to the first quarter $(p=0.001)$. Similar trends were shown for a decrement in HSRD during the second $(\mathrm{ES}=0.45)$, third $(\mathrm{ES}=0.78)$, and fourth $(\mathrm{ES}=1.23)$ quarters of play when compared to the first quarter $(p=$ $0.005)$. Additionally, $S D$ was significantly reduced across all quarters (ES: $0.85 ; 1.23 ; 1.45)$ when compared to the first quarter $(p=0.002)$. Finally, decrements in the number of accelerations completed were seen for the second $(\mathrm{ES}=$ $0.55)$, third $(\mathrm{ES}=0.88)$, and fourth $(\mathrm{ES}=1.53)$ quarters of play when compared to the first quarter. Figure 1 shows the temporal changes in HSRD across quarters during match-play.

A significant effect for position $(F=5.21, p<0.001)$ with regard to decrement in running performance revealed that midfielders had a greater TD decrement when compared to all other positions ( $\mathrm{ES}=0.35-0.89$ ). Half-backs experienced significantly $(p=0.002)$ higher decrement in TD when compared to full-forwards $(\mathrm{ES}=1.35)$ and full-backs $(\mathrm{ES}=1.16)$. Similar effects for position $(F=8.81, p<0.001)$ revealed that midfielders had a greater HSRD decrement when compared to all other positions ( $\mathrm{ES}=0.82-2.39)$. Half-forwards experienced higher decrement in HSRD when compared to fullforwards $(E S=1.35)$ and full-backs $(E S=1.16)$ only. Figure 2 shows the decrement in HSRD with respect to position. Significant main effects for position on $S D$ decrements were revealed $(F=6.11, p<0.001)$, with half-backs and midfielders having higher $S D$ decrement when compared to other positions ( $E S=0.92-2.19$ ). Post hoc analyses identified that midfielders had a greater acceleration decrements when compared to all other positions $(\mathrm{ES}=0.42-1.39)$. Halfforwards experienced higher decrements in accelerations when compared to full-forwards $(\mathrm{ES}=0.85)$ and full-backs $(\mathrm{ES}=1.16)$ only. Interestingly, no main effects were observed for playing position for peak speed between quarters of match-play.

\section{Discussion}

The current study is the first to describe the within-match variation of running performance in elite-level Gaelic football. Results show that the running performance of Gaelic football players changes as the match progresses (Table 1). Specifically, there were reductions in TD completed after the first quarter of match-play, with additional decrements seen across all quarters of match-play $(-3.8$ to $-5.9 \%)$. Furthermore, significant decrements were observed for HSRD across the quarters of match-play for all positions $(-8.8$ to $-19.8 \%)$. Interestingly, there was a noted reduction in the number of accelerations completed, but no change in peak speeds achieved by players. Finally, the study has identified a specific running profile for elite Gaelic football match-play.

The current analysis revealed that midfielders followed by half-forwards and half-backs covered the greatest distance across all speed determined thresholds during match-play. Further positional comparisons showed that these 3 positional groups experienced the highest decrements in running performance across the quarters of match-play (Table 1). The differences in running performance across positions may be explained by the specific tactical roles of each playing position, which can allow for greater a freedom to complete higher distances and consequently accrue higher decrements in running performance during competitive match-play. Overall, the results show that higher running performance during the early periods of competition may influence subsequent running performance toward the end of competitive match-play.

The running performance of players highlighted in this study is similar to previous investigations $(7,14)$, with results reported showing similar distances being covered during match-play. However, we are the first to report significant running performance decrements across all quarters of match-play, which is in contrast previous investigations conducted in Gaelic football $(15,20)$. Additionally, significant positional differences in all running variables were observed during match-play. Unfortunately, previous observations have used visual and audio time-motion-based analysis to estimate the activity profile of players and therefore caution must be taken in comparing the result because of several limitations including validity and reliability of the data entry procedure $(14,20)$. Additionally, the age profile of previous data also questions the relevance of previous findings to coaches within the confines of the modern game. Teams are now employing more sophisticated tactics, which have been shown to impact running performance variables in other team sports (21). Furthermore, year to year changes in overall and positional physical performance have been 
reported in Australian Rules football (27) and soccer $(1,3,4,6)$. The data reported in the current investigation must be considered in conjunction with the teams investigated. The current investigation sample comprised of division 1 and division 2 teams; as such the divisional ranking may explain the increased running performance seen in relation to previous investigations (14). It has been shown that players from less successful soccer teams complete higher activity levels than there more successful counterparts, but spend less time in possession of that ball and are less technically proficient $(3,22)$. Future studies should examine if such relationships exist in elite Gaelic football.

The running performance observed in the current study demonstrate that Gaelic football players spend less time completing high-speed running actions as the match progresses with consistent reductions in performance observed across quarters of play. Interestingly, similar trends were observed for total and sprint distances across the duration of match-play. These results are in agreement with previous studies in Australian rules football $(8,9)$, rugby league (24), and soccer $(4,19,22)$ that report running performance decrements across quarters of play. It is unclear if the decrement in performance is related to fatigue. It has previously been suggested that game players may alter their running performance profile during intermittent activity on the basis of prematch contexts, fitness levels, match importance and physiological alterations during play, which may explain the within match variation observed for elite-level matchplay (11). Additionally, previous investigations of injury occurrences in elite Gaelic football (26) have highlighted an increase in the likelihood of injury as the game progresses; interestingly, this investigation showed 56.9\% (95\% CI, 46.167.1) of injuries were experienced in the second half as opposed to $39.7 \%(95 \%$ CI, 29.8-50.5) in the first half. In addition, the fourth quarter of the game had the most injuries $(29.3 \%, 95 \%$ CI, 19.2-42), whether the occurrence levels can be related to the decrement in performance is yet to be fully understood and merits future investigation.

The current investigation has highlighted between position differences in running performance decrement (TD, HSRD, SD, and accelerations) across quarters of match-play. The present results demonstrate that the middle 3 positions (half-back, midfield, and half-forward) complete higher volumes of running in all quarters of match-play. Specifically, midfielders covered greater TD compared with other playing positions also HSRD was greater for midfielders and half-forwards and half-backs in comparison with fullforwards and full-backs. The current results are comparable to those that have been reported previously during Australian rules football competition play where a temporal change in TD, HSRD, and SD was observed $(8,9)$. Results indicate that both HSRD and SD are reduced significantly in the second, third, and fourth quarters of match-play. The decrement in running performance was position specific with the middle 3 positional roles exhibiting the highest decrement across high-speed running performance variables. Midfield players $(20 \%)$ experienced the highest decrement in highspeed running performance across quarters of play followed by half-back (15.3\%) and half-forward lines (12.2\%). A nonsignificant decrement was observed for both full-back (5.6\%) and full-forward lines (5.5\%). Similar trends were observed for sprint running performance decrements with greater decrements for midfielders and half-forwards and half-backs in comparison with full-forwards and full-backs. A reduction in running performance has been previously reported during elite-level Australian football matches $(8,9)$ and attributed to a reduction in glycogen stores (25). These findings may suggest a need for position-specific nutritional resupplementation strategies during match-play and at half time. The current findings are similar to those observed in Australian rules football (9) and soccer $(19,22)$. The reported decrement could potentially be explained by several factors such as tactical systems, positional roles, opposition played, or fitness levels (21). Regardless of the above, the results have practical implications for coaches deciding when to make player interchanges and which player to interchange during match-play.

With technical, tactical and physical activity profiles during match-play being inextricably linked in the football codes $(1,18,22,24)$, a limitation of the current study is that technical skill efficiency and the tactical approach of the teams analyzed could not be controlled for. Future studies should examine the complex relationships between these factors, so that the relationships of running performance to overall Gaelic football performance are better understood. With previous investigations reporting that running performance in team sports is increasing annually $(6,27)$, there is a need for practitioners to fully understand how the running performance of Gaelic football players is developing and changing year on year. Therefore, we recommend that the yearly change in running performance be reported for elite Gaelic football players.

\section{Practical Applications}

To provide relevant data on match-play, the current study examined the match running performance of elite Gaelic football players. The present study shows that higher exercise intensities during the early periods of competition in Gaelic football may influence the decrements in running performance toward the end of match-play. The results show that the first quarter of match-play is the most intense, and that in subsequent quarters, there is a reduction in TD, HSRD, SD, and accelerations. The reductions seen in running-based measures appear to be position specific with midfielders followed by half-backs and half-forwards experiencing the highest decrements in running measures when compared to other positions. This highlights the need for specific nutritional interventions related to positional role to be employed within match-play and at half time to best counteract these decreases in running performance. With 
training specificity an important consideration for coaches to stimulate training adaptations to improve match-play performance, the study has shown that each position has a distinct running profile that needs to be considered in preparing teams for competition. Coaches and applied practitioners should consider the positional running profiles of players when developing training drills to better optimize training outcomes for match-play performance.

\section{Acknowledgments}

The authors of the present study would like to thank all the players and teams who participated during the research period. No external sources of funding were provided for this study. The authors have no relevant conflicts of interest to declare.

\section{REFERENCES}

1. Barnes, C, Archer, DT, Hogg, B, Bush, M, and Bradley, PS. The evolution of physical and technical performance parameters in the English Premier League. Int J Sports Med 35: 1-6, 2014.

2. Beasley, KJ. Nutrition and Gaelic football: Review, recommendations and future considerations. Int J Sport Nutr Exerc Metab 25: 1-13, 2015.

3. Bradley, PS, Archer, DT, Hogg, B, Schuth, G, Bush, M, Carling, C, and Barnes, C. Tier-specific evolution of match performance characteristic in the English Premier League: It's getting tougher at the top. J Sports Sci 11: 1-8, 2015.

4. Bradley, PS, Sheldon, W, Wooster, B, Olsen, P, Boanas, P, and Krustrup, P. High-intensity running in FA Premier League soccer matches. J Sports Sci 27: 159-168, 2009.

5. Buchheit, M, Allen, A, Poon, TK, Mondonutti, M, Gregson, W, and Di Salvo, V. Integrating different tracking systems in football: Multiple camera semi-automatic system, local positioning measurement and GPS technologies. J Sports Sci 32: 1844-1857, 2014.

6. Bush, M, Barnes, C, Archer, DT, Hogg, B, and Bradley, PS Evolution of match performance parameters for various playing positions in the English Premier League. Hum Mov Sci 39: 1-11, 2015.

7. Collins, DK, Solan, B, and Doran, DA. A preliminary investigation into high-intensity activity during elite Gaelic football. J Sports Ther 1: 10, 2013.

8. Coutts, AJ, Kempton, T, Sullivan, C, Bilsborough, J, Cordy, J, and Rampinini, E. Metabolic power and energetic costs of professional Australian football match play. J Sci Med Sport 18: 219-224, 2015.

9. Coutts, AJ, Quinn, J, Hocking, J, Castagna, C, and Rampinini, E. Match running performance in elite Australian Rules football. $J$ Sci Med Sport 13: 543-548, 2010.

10. Duffield, R, Reid, M, Baker, J, and Spratford, W. Accuracy and reliability of GPS devices for measurement of movement patterns in confined spaces for court-based sports. J Sci Med Sport 13: 523-525, 2009.

11. Edwards, AM and Noakes, TD. Dehydration: Cause of fatigue or sign of pacing in elite soccer? Sports Med 39: 1-13, 2009.

12. Hopkins, W, Marshall, S, Batterham, A, and Hanin, J. Progressive statistics for studies in sports medicine and exercise science. Med Sci Sports Exerc 41: 3-12, 2009.

13. Jennings, D, Cormack, S, Coutts, AJ, Boyd, LJ, and Aughey, RJ. Variability of GPS units for measuring distance in team sport movements. Int J Sports Physiol Perform 5: 565-569, 2010.

14. Keane, S, Reilly, T, and Hughes, M. Analysis of work rates in Gaelic football. Aus J Sci Med Sport 25: 100-102, 1993.

15. Maddison, R and Ni Mhurchu, C. Global positioning system: A new opportunity in physical activity measurement. Int J Behav Nutr Phys Act 6: 73, 2009.

16. Malone, S, Collins, DK, McRobert, AP, Morton, J, and Doran, DA. Accuracy and reliability of VXsport global positioning system in intermittent activity. Presented at: The 19th Annual Congress of the European College of Sport Science, July 5, 2014; Amsterdam, the Netherlands.

17. Malone, S, Solan, B, Collins, DK, and Doran, DA. The positional match running performance of elite Gaelic football. J Strength Cond Res 30: 2292-2298, 2016.

18. McErlean, CA, Cassidy, J, and O'Donoghue, PG. Time-motion analysis of gender and positional effects on work-rate in elite Gaelic football competition. J Hum Mov Stud 38: 269-286, 2000.

19. Mohr, M, Krustrup, $P$, and Bangsbo, J. Match performance of highstandard soccer players with special reference to development of fatigue. J Sports Sci 21: 519-528, 2003.

20. O'Donoghue, $\mathrm{P}$ and King, S. Activity profile of men's Gaelic football. In: Science and Football $V$. T Reilly, ed. Oxford, United Kingdom: Routledge, 2000. pp. 207.

21. Paul, DJ, Bradley, PS, and Nassis, GP. Factor affecting running performance in elite soccer players: Shedding some light on the complexity. Int J Sports Physiol Perform 10: 516-519, 2015.

22. Rampinini, E, Impellizzeri, FM, Castagna, C, Coutts, AJ, and Wisloff, U. Technical performance during soccer matches of the Italian Serie A league: Effect of fatigue and competitive level. $J S c i$ Med Sport 12: 227-233, 2009.

23. Reilly, T and Collins, K. Science and the Gaelic sports: Gaelic football and hurling. Euro J Sport Sci 8: 231-240, 2008.

24. Sirotic, AC, Coutts, AJ, Knowles, H, and Catterick, C. A comparison of match demands between elite and semi-elite rugby league competition. J Sports Sci 27: 203-211, 2009.

25. Williams, C and Rollo, I. Carbohydrate nutrition and team sport performance. Sports Med 9: 1-10, 2015.

26. Wilson, F, Caffrey, S, King, E, Casey, K, and Gissane, C. A 6-month prospective study of injury in Gaelic football. BrJ Sports Med 41 317-321, 2007.

27. Wisbey, B, Montgomery, PG, Pyne, DB, and Rattray, B. Quantifying movement demands in AFL football using GPS tracking. J Sci Med Sport 13: 531-536, 2010. 